\title{
A Mobility Model of Theme Park Visitors
}

\author{
Gürkan Solmaz, Student Member, IEEE, Mustafa İlhan Akbaş, Member, IEEE, \\ and Damla Turgut, Member, IEEE
}

\begin{abstract}
Realistic human mobility modeling is critical for accurate performance evaluation of mobile wireless networks. Movements of visitors in theme parks affect the performance of systems which are designed for various purposes including urban sensing and crowd management. Previously proposed human mobility models are mostly generic while some of them focus on daily movements of people in urban areas. Theme parks, however, have unique characteristics in terms of very limited use of vehicles, crowd's social behavior, and attractions. Human mobility is strongly tied to the locations of attractions and is synchronized with major entertainment events. Hence, realistic human mobility models must be developed with the specific scenario in mind. In this paper, we present a novel model for human mobility in theme parks. In our model, the nondeterminism of movement decisions of visitors is combined with deterministic behavior of attractions in a theme park. The attractions are categorized as rides, restaurants, and live shows. The time spent at these attractions are computed using queueing-theoretic models. The realism of the model is evaluated through extensive simulations and compared with the mobility models SLAW, RWP and the GPS traces of theme park visitors. The results show that our proposed model provides a better match to the real-world data compared to the existing models.
\end{abstract}

Index Terms—Mobility model, human mobility, wireless network, theme park.

\section{INTRODUCTION}

Recent advances in mobile devices enabled the increased popularity and usage of mobile applications. Urban sensing applications, where mostly smart phones are used, and wireless sensor networks with mobile sinks are examples of these applications. The realistic modeling of human movement has significant importance for the performance assessment of such mobile wireless systems.

Human mobility models simulate the movement patterns of the mobile users and they form a key component of the simulation-based performance evaluation [1]. Early mobility models relied on some type of variations of the idea of a random walk. Examples of this approach include the random waypoint (RWP) [2] and Brownian Motion [3] models. These models are only very coarse approximations of human behavior. One of the most important characteristics of human mobility is the combination of regularity and spontaneity in deciding the next destination. This behavior can also be defined as making both deterministic and nondeterministic decisions in the same time period. Considering the theme park scenario, visitors usually preplan their visit. They try to optimize their time on rides while minimizing the time to walk from one attraction to another. Nevertheless, when they are in the park, they may change their decisions spontaneously depending on various factors. Random mobility models such as RWP do not provide a good match for this behavior.

The current human mobility models can be classified into two groups as trace-based [4] and synthetic [5] models. The trace-based models generally use GPS traces and Bluetooth connectivity observations. However, it is difficult to

- The authors are with the Department of Electrical Engineering and Computer Science, University of Central Florida, Orlando, FL, 32816. E-mail: \{gsolmaz,miakbas,turgut\}@eecs.ucf.edu collect real data and the amount of publicly available data is limited. Therefore, synthetic models, which are defined on mathematical basis, are widely used in simulations. Most mobility models aim for a generic human movement modeling. However, different areas exhibit distinct human mobility patterns, such as the areas of different cities [6]. The mobility decisions of people are driven by their goals in their environments. For instance, walks in a city center are driven by the need for rapidly reaching to specific destinations. In a university campus, walks are constrained in space by the destination of classrooms, meeting rooms, and cafeterias. At the same time, they are constrained in time by the schedules of classes and meetings. In an amusement park, on the other hand, the movement would be determined by the attractions planned to be visited. These examples illustrate the need for the scenario-specific modeling of human mobility.

Theme parks are large crowded areas with unique characteristics in terms of movement patterns of visitors, attractions in various locations and walking paths connecting the attractions. In this paper, we present a mobility model of theme park visitors. The outputs of the model are the synthetic movement tracks, pausing locations (waiting points) and pausing times [7]. First, the fractal points are generated by the model in order to create the pausing locations. The concentrated locations of these fractal points are defined as the meeting locations of visitors or attractions. This method decreases the number of waypoints in a map, allowing the simulation of large numbers of visitors. It also makes the mobility model more realistic since real attractions such as restaurants or rides in the environment can be simulated by their individual models. These locations are grouped into four main attraction types of theme parks: main rides (RD), medium-sized rides (M-RD), live shows (LS), and restaurants (RT). The waiting times of visitors at these attractions are modeled using queueing theory. Moreover, we define 
walking areas of visitors as landmarks in the theme park in order to separate the walking paths from the roads on which transportation vehicles are used.

Let us now consider how such a model is useful for wireless mobile applications. For instance, a wireless sensor network (WSN) can be deployed in a theme park for finding the fastest way to move from one location to another considering the current density of the crowds in different areas of the park ([8], [9], [10]). Such a WSN would rely on the personal mobile devices of the visitors and can be used to offer an interactive theme park experience. Social networking applications or multi-player games can be offered to the visitors with the support of a deployed wireless system or in an ad hoc working mode. The performance of such a system would highly depend on the mobility of the users and must be evaluated by simulations before deployment.

Another class of applications would be the theme park administration. Theme park administrators must direct visitors efficiently among attractions and balance the number of visitors at each attraction. It is desirable to balance the density of the crowd in different areas of the park due to efficiency of the attractions as well as the security of the visitors. The administrators can use the mobility model to estimate the possible impact of their decisions such as the distribution of live entertainers or the arrangement of the paths for pedestrian traffic. The predictive results of the model can be used to decide the locations of security personnel. The mobility model is also used as a base for disaster simulations and emergency management applications [11].

In this paper, we present a mobility model of theme park visitors considering the nondeterministic macro-mobility decisions of the visitors as well as the deterministic behaviors of the attractions. Our model is successful in terms of representing the social behavior of people to gather in attractions, spending time in queues, and movement decisions in terms of the least-action principle of human walks. The outcomes of the proposed model are synthetically generated mobility traces. These mobility traces are compared to the real-life theme park GPS traces and the traces of two mobility models. The mobility traces of our model have the best statistical match to the GPS traces amongst the tested synthetic models in terms of flight length distributions, average number of waiting points, and waiting times.

The rest of the paper is organized as follows. We provide a detailed description for our mobility model in Section 2 . We evaluate the validity of the model in Section 3, summarize the related work in Section 4 and finally conclude in Section 5 .

\section{Human Mobility Model}

In this section, we present the scenario-specific mobility model for the theme park visitors. Before describing the model, let us briefly explain the fundamental characteristics of these entertainment areas. Theme parks are large areas with one or more "themed" landmarks that consist of attractions. Visitors of a landmark plan to see a subset of these attractions by walking during their scheduled visit. SLAW [12] model provides an effective strategy in representing social contexts of common gathering places of pedestrians by fractal points and heavy-tail flights on top of

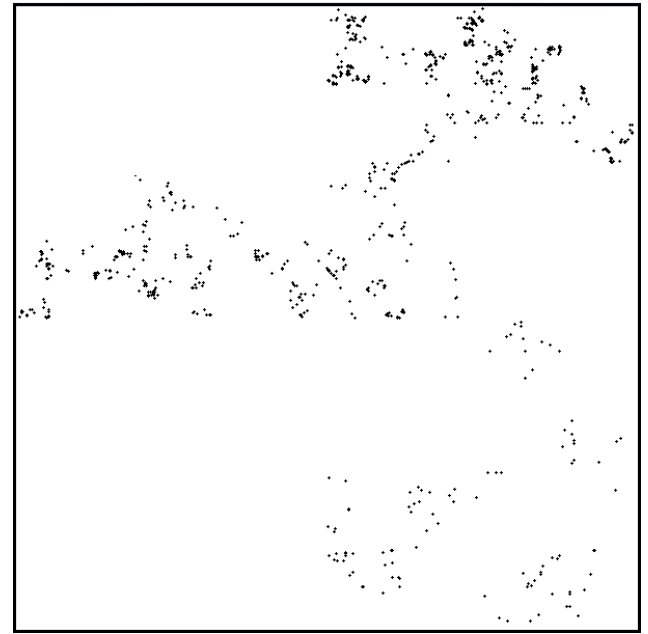

Fig. 1. Fractal point generation phase of the model.

these fractal points. We extend this idea for a more realistic mobility model and apply queueing models to represent the behavior and effects of different types of attractions on the mobility of theme park visitors.

\subsection{Modeling a theme park}

The modeling of a theme park consists of five main phases, which starts with the first phase of fractal points generation, and ends with the theme park model.

\subsubsection{Fractal points}

We use the term fractal points based on its usage in the SLAW mobility model. In our model, the fractal points are initially created in an empty area using the fractional Gaussian noise or Brownian Motion generation technique (fGn or fBm), as described by Rhee et al. [13]. A fractal point can be considered as a waypoint at the beginning. All fractal points and the area, in which these points are generated in, are used to form a landmark as described in the following phases. It is shown that the use of fractal points and leastaction trip planning on top of these self-similar points satisfy fundamental statistical features of human walk [12]. As a human behavior, people are more attracted to visit popular places. This characteristic of human mobility can be expressed using fractal points as explained in the next subsection. Fig. 1 demonstrates the first phase of the model in a scenario, where 1000 fractal points are generated in an area of 1000x1000 meters.

\subsubsection{Clusters}

After generation of the fractal points, we determine the parts of the area with highest density of the points. The goal of this phase is finding the popular areas, where people are more attracted to gather.

We use a modified version of DBScan [14] algorithm on the generated fractal points to find the attraction locations. DBScan is a density based clustering algorithm for discovering clusters with noise points, which has two input parameters, epsilon Eps and minimum number of points (neighbors) MinPts. 


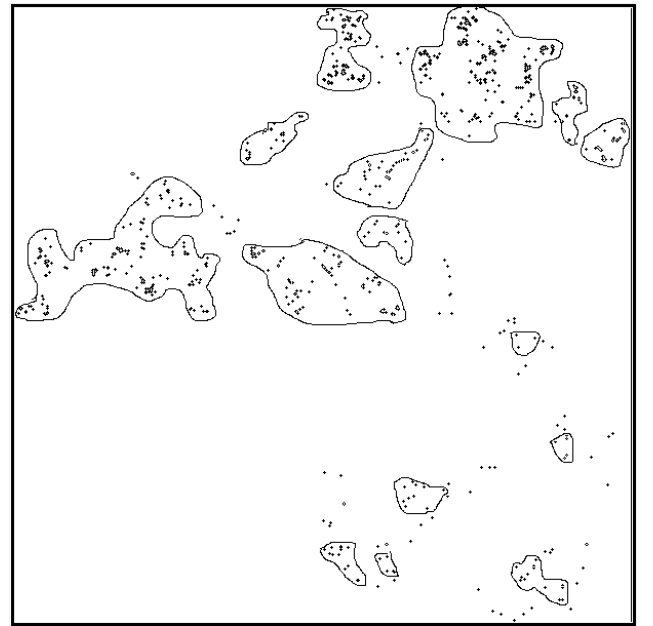

Fig. 2. Clusters generated by DBScan over 1000 fractal points.

The Eps-neighborhood of a point $p$, denoted by $N_{E p s}(p)$, is defined by $N_{E p s}(p)=\{q \in D \mid \operatorname{dist}(p, q) \leq E p s\}$, where $D$ is the database of points. In our DBScan approach, for each point in a cluster, there are at least MinPts neighbors in the Eps-neighborhood of that point.

In our model, DBScan algorithm is modified based on the requirements of the scenario. The input parameters include epsilon, minimum number of neighbors, number of clusters and proportion of noise points among all fractal points. The number of clusters and noise point ratio are used to specify a landmark. For instance, if there are 25 attractions in a theme park, the number of clusters becomes 25 . The non-clustered point ratio is used to determine the nondeterminism in mobility empirically (e.g. $10 \%$ ) or based on statistical data collected from the visitors of a theme park. The values of the minimum number of neighbors and epsilon are iteratively searched with a heuristic, which alters the values of these two parameters according to the results of the previous iteration. This heuristic is based on the fact that changing the values of these two parameters directly changes the resulting number of clusters and the non-clustered point ratio. For instance, if epsilon has a larger value and minimum number of neighbors has a smaller value, DBScan produces less number of clusters, with a smaller non-clustered point ratio.

Let us assume a landmark is required to have 10 clusters and the proportion of non-clustered points to be approximately 0.10 . The initial epsilon and minimum number of neighbors are set as 30 meters (for dimensions of 1000x1000 meters) and 8 empirically. After setting the initial values, the fractal points are scanned iteratively to set the new values for epsilon and the number of neighbors parameters. When the expected number of attractions and the expected approximate proportion of non-clustered points are achieved, the clustering of fractal points are finalized.

The clustering of the fractal points determines the areas with highest densities of fractal points. Fig. 2 shows an example clustering output with over 1000 fractal points in an area of $1000 \times 1000$ meters. In this example, 15 clusters are generated and marked, whereas $10 \%$ of the fractal points are not included in the clusters.

\subsubsection{Attractions and noise points}

The clusters as the regions with highest number of fractal points and non-clustered points are obtained from the previous step. In this phase, the most dense areas found by clustering step are marked as "attractions".

In our model, attractions are represented by queueing models. We decide on the types and the weights of the queues based on the number of fractal points and the previous work on theme park design [7]. The weight of a queue is defined according to the number of fractal points included in its corresponding cluster. The central point of a queue is the average position of all the fractal points included in its corresponding cluster. Non-clustered fractal points are marked as "noise points". Wanhill [15] defined the attractions in a theme park by queueing models and the specified expected percentages are given in Table 1.

TABLE 1

Attraction percentages

\begin{tabular}{|l|l|l|}
\hline Attraction & Queue model & Percentage \\
\hline Main rides (RD) & $\mathrm{M} / \mathrm{D} / \mathrm{n}$ & $17 \%$ \\
\hline Medium-size rides (M-RD) & $\mathrm{M} / \mathrm{D} / \mathrm{n}$ & $56 \%$ \\
\hline Restaurants (RT) & $\mathrm{M} / \mathrm{M} / 1$ & $17 \%$ \\
\hline Live shows (LS) & $\mathrm{M} / \mathrm{M} / \mathrm{n}$ & $10 \%$ \\
\hline
\end{tabular}

Each attraction has a corresponding queue according to its particular properties. $M / D / n$ queue has a constant service time, whereas $M / M / 1$ and $M / M / n$ queues have service times according to the exponential distribution. The number of service channels $n$ corresponds to the amount of visitors served per service time. In our model, $M / D / n$ queue model is used for the main rides and the mediumsized rides since they have similar queue behaviors. $M / M / 1$ queue model is used for restaurants and $M / M / n$ queue model is employed for live shows since restaurants and live shows have exponential service rates, while the service rates of main rides and medium-sized rides are constant.

The waiting points for the visitors in a landmark are defined by the locations of the attractions or the noise points. The attractions are clusters of fractals, whereas the noise points are non-clustered fractals. Both can be considered as the locations where the visitors spend a certain amount of time. For example, a point where a visitor stops for a while to take pictures can be considered as a noise point in the scenario.

\subsubsection{Landmarks}

Landmarks are generated as a result of the previous steps, including the generation of fractal points, density-based iterative clustering, generation of queues according to their weights, queue types, and service rates. In this phase, we form landmarks by the inclusion of visitors, which are mobile elements of a landmark. A specified number of visitors are distributed to attractions and noise points randomly. The random distribution is achieved according to the weights of attractions, and the weights of the noise points are set to 1 .

A landmark is a place where there are multiple static queues, static noise points, and mobile visitors. Each landmark has two dimensions specifying its size. Fig. 3 shows a landmark model with initial placement of 20 visitors (mobile nodes), queues, and noise points in an area of 


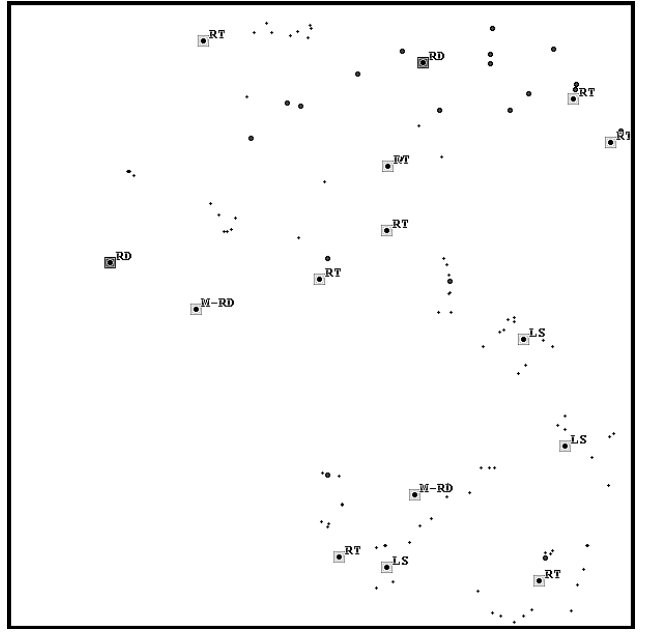

Fig. 3. A landmark model including attractions, noise points and initially distributed mobile nodes.

1000x1000 meters. In this figure, central points of the queues are represented by squares. The noise points and initially located mobile nodes are shown by small dots and circles respectively. Each queue is presented with its attraction type: main rides $(\mathrm{RD})$, medium-sized rides $(\mathrm{M}-\mathrm{RD})$, live shows (LS), and restaurants (RT).

The proposed landmark model is used to model walking areas of the visitors such as the Magic Kingdom park (landmark) in the Disney World theme park by assigning the number of queues and the proportion of noise points accordingly. The landmark model separates walking areas of visitors from the areas where the vehicles are used for transportation. This differentiation is important for the realistic mobility modeling of visitors in large theme parks, where walking between various landmarks is not possible due to the long distances.

\subsubsection{Theme Park Map}

For modeling the theme park, we use a graph theoretical approach. The theme park map is modeled as a graph consisting of vertices and weighted non-directional edges. Each vertex in the graph represents a landmark. If there is a road between two landmarks, an edge is added with a weight corresponding to the transportation time.

Theme parks are usually large areas with transportation services among the main locations of attractions such as buses, trains and cars. Most of the theme parks are located in non-uniform 2D areas, which brings a challenge to simulate a theme park with a model assuming a uniform 2D area. By separating landmarks as vertices in a theme park graph model and adding weighted non-directional edges between the landmarks, we generalize the model of human mobility in a landmark to the human mobility in the whole theme park. The mobility model includes the landmarks and the edges between them for transportation of visitors. We do not assume that a theme park is a uniform $2 \mathrm{D}$ area, since it includes geographical obstacles such as areas without pavements for pedestrians and paths or roads used for transportation. These characteristics enable our mobility model to be more realistic compared to the existing models.
Fig. 4 illustrates the phases of our model in a stepby-step fashion. These phases start with the generation of fractal points, density-based clustering and continues with the generation of the attractions and the noise points. The attractions, the noise points and the visitors all together form a landmark as shown in the fourth phase. In the last phase, multiple landmarks and roads are modeled with a graph.

\subsection{Visitor model}

In the model, the visitors are represented by mobile nodes. We define the states of the mobile nodes in a landmark as "initial", "inQueue", "moving", "inNoisePoint" and "removed". At the beginning of the simulation, all mobile nodes are in "initial" state. A mobile node changes its state to "inQueue" when it starts waiting in a queue. The state changes to "inNoisePoint" when the node starts waiting in a noise point. There are two different states of waiting in order to differentiate waiting in a noise point or in a queue. When a mobile node starts changing its location to arrive to a new destination, which may be an attraction or a noise point in the landmark, it is in the "moving" state. The state of a mobile node is "removed" when the hangout time of the node passes. Fig. 5 shows the five states of visitors and the state transitions.

Initially, each visitor decides on the amount of time to stay in the particular landmark, which is defined as the hangout time. Hangout times of the visitors are generated by using the exponential distribution. Then, each visitor selects a subset from the set of all attractions at the landmark to visit. The size of the subset (the number of queues to visit) selected by a visitor is proportional to the corresponding hangout time of that visitor. If the visitor is not in "inQueue" state when the hangout time ends, the visitor leaves the landmark. In other words, the visitor arrives at an exit point of the landmark. If the visitor is waiting in a queue, (in "inQueue" state), the visitor continues to wait in the queue and leaves the landmark after being serviced. We assume every visitor has a constant speed. After attraction subset decision, the visitors move according to the least action principle among the selected attractions and noise points. The visitor marks an attraction or a noise point as visited and does not visit these points later. This principle is also used to explain how people make their walking trails in public parks

The next destinations of visitors are decided by using Algorithm 1, which is a modified version of Least Action Trip Planning (LATP) [12] algorithm. In LATP, a visitor tries to minimize the Euclidean distance traveled from a waiting point to a new waiting point (destination). The waiting points are either the attractions or the noise points in the landmark. This strategy is different than Dijkstra's Shortest Path since it does not always cause the new destination to be the nearest waiting point, where every unvisited point has a probability to be the next destination. The parameter $\alpha$ is used to determine this probability. The algorithm is modified to match the requirements of our mobility model. In Algorithm 1, $A$ is the set of attractions which are planned to be visited by the visitor, while $N$ is the set of all noise points. $W$ represents the set of attraction weights, while 


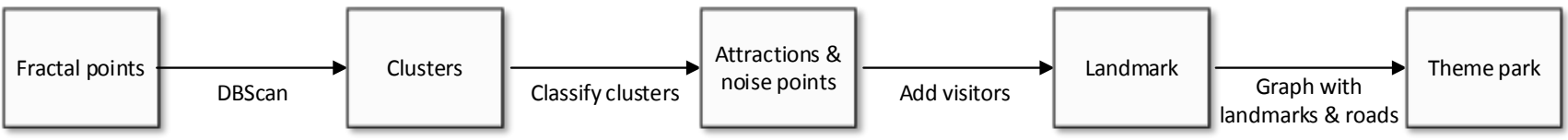

Fig. 4. The phases of modeling a theme park.

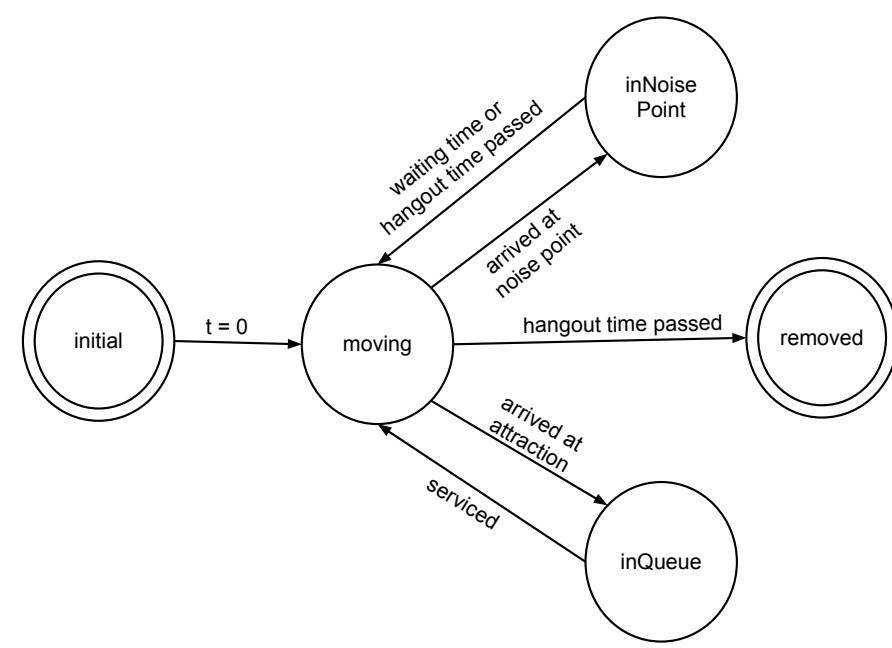

Fig. 5. States of a visitor.

$c p$ is the current position of the visitor. $\mathrm{Pr}$ is the set of probabilities of the next destination points of the visitor. Waiting points are not identical and have varied weight values, while the weight of a noise point is always 1 and the weight of a queue (attraction) is set as the number of fractal points included in its corresponding cluster. The probabilities of the queues with larger weight values, such as main rides, are higher for selection as the new destination points. In other words, visitors are more attracted to gather in queues with larger weight values. For the calculation of Euclidean distances $(d(c p, a)$ for attractions and $d(c p, n)$ for noise points), we use the exact positions of the noise points in the landmark and the positions of the central points of queues.

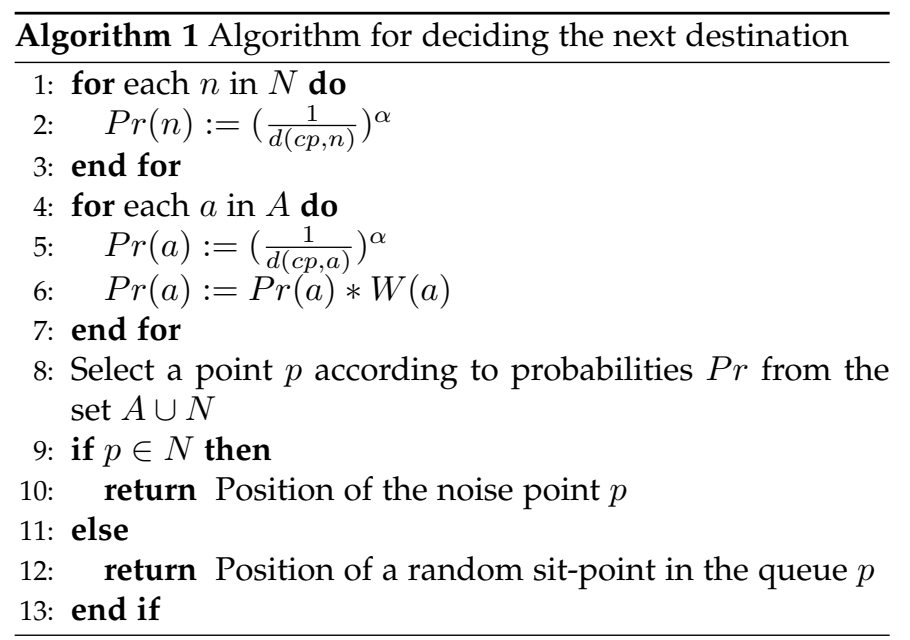

At each iteration of the simulation, we check the queues to find the number of visitors serviced and the states of all visitors for possible changes. For instance, if a visitor is serviced by an attraction, the state of the visitor must change from "inQueue" to "moving".

When an attraction is selected, the visitor goes to a random sit-point inside the clustered area as the new destination position. Waiting time of a visitor in a queue depends on the number of visitors already waiting in the queue ahead of that visitor, service rate, and the number of visitors per service of the attraction. When a visitor goes to a noise point, the waiting time of the visitor is generated using the truncated Pareto distribution.

Most theme park visitors travel in groups such as families. While this model is based on individual mobility decisions, group mobility characteristics of the proposed approach or the social behaviors of the groups formed as a result of the attractions can be analyzed to improve the mobility model.

\subsection{Theme park with multiple landmarks}

The mobility model can be easily applied to model a complete theme park scenario. Each smaller park in a large theme park would be modeled as a landmark. For each park, real dimension lengths are used to specify the 2D rectangle area of a landmark. OpenStreetMap [16] can be used to determine the sizes of the theme parks.

In the real scenario, the number of attractions and types of those attractions are generally known; however, if this is not the case, the queue types of the attractions and the numbers specified in this paper can potentially be used. With this approach, a portion of a theme park can be modeled as a landmark.

Each visitor in this scenario has a total hangout time, which is the amount of time to spend in the theme park. Initially, visitors decide on the parks (landmarks) to visit in an order such that the transportation (minimum weights) between them is minimized. A visitor also plans to visit particular attractions when entering a new landmark. After finishing the hanging out time in the park, the visitor goes to the next planned park through the road connecting the two parks.

Fig. 6 contains three main parks (landmarks) of the Disney World theme park in Orlando; namely, Epcot, Animal Kingdom and Hollywood Studios. OpenStreetMap [16] is used to illustrate the model on this map and the Magic Kingdom park is not included here for illustration purposes. In this figure, landmarks are the vertices and the lines connecting landmarks are the edges with different weights. As you can see, the landmarks have labels $\mathrm{L}_{1}, \mathrm{~L}_{2}$ and $\mathrm{L}_{3}$, and the weights of the edges between them have labels $W_{1}, W_{2}$ and $\mathrm{W}_{3}$. The landmarks can be generated according to the 


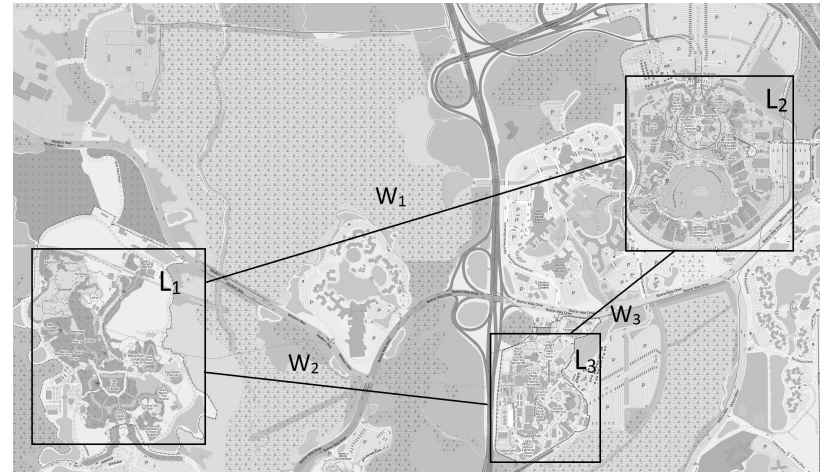

Fig. 6. An illustration of the application of model to a real-world scenario: Disney World parks in Orlando.

actual sizes of the areas of the main parks, and the weights are set with the actual transportation times. Dimensions and numbers of attractions are also set for each park. The simulation of the model is applied to the real scenario by this graph theoretical approach, which generates realistic synthetic traces of human mobility for the theme parks included in the scenario.

\section{Simulation Study}

\subsection{Simulation environment and metrics}

In this section, the experiments are carried out to validate our mobility model in landmarks and observe the effects of the unique parameters of the model. The simulation of our model generates synthetic mobility traces of visitors in a $2 \mathrm{D}$ terrain. The terrain is specified by dimensions, number of attractions and the noise point ratio. Fig. 7 shows an output example of a simulation run with 20 mobile nodes, which is taken when simulation time is 3600 seconds.

Mobile nodes in the simulation draw their trajectory lines while moving. These trajectory lines are the consecutive points in the figure, which illustrate the movement of the mobile nodes in the landmark. The waiting points are the points of intersections of consecutive trajectory lines. The waiting points are either noise points or they are located inside the attractions. Fig. 8 demonstrates another simulation with 200 mobile nodes after 3600 seconds of simulation time. In this figure, by looking at the positions of the mobile nodes represented by small circles, one can observe the expected human mobility behavior to gather in common places, which are the dense regions of fractal points in the model. The use of fractal points and planning trips according to the least action principle represent this social behavior.

We conducted simulations for square landmarks with dimensions of 1000x1000 meters and 2000 mobile nodes. For all experiments, total simulation time is 10 hours with a sampling time of 10 seconds. Mobile nodes have hangout times that are exponentially distributed between 2 hours and 10 hours. For a 1000x1000 landmark, we used 15 queues and approximately $10 \%$ noise point ratio. Then we changed these parameter values to observe their effects on the metrics.

We considered three metrics throughout the simulations: 1) flight length that specifies the distance between a pair of

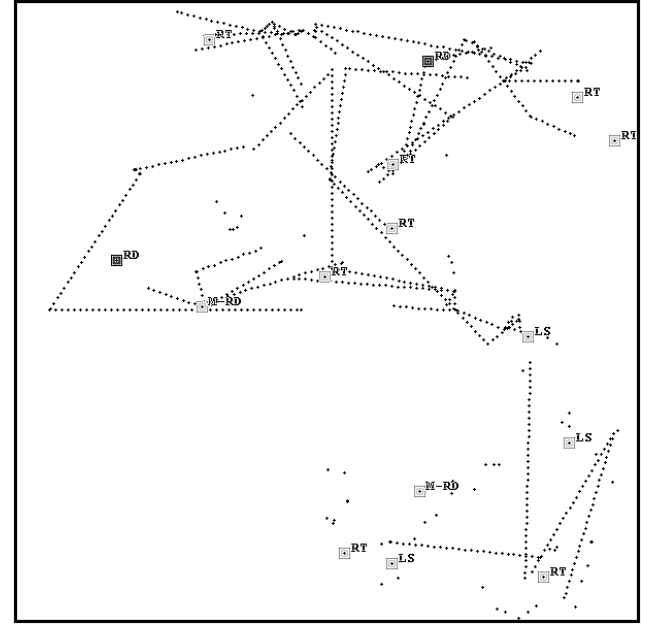

Fig. 7. Trajectories of 20 mobile nodes after 1 hour simulation time.

consecutive waiting points of a mobile node; 2 ) number of waiting points that analyzes the wait frequency of a mobile node in a time interval and; 3 ) waiting time of visitors that specifies the time spent by a mobile node at noise points or inside attractions.

Attractions have two parameters: (expected) service time and number of service channels that represent the number of visitors leaving the attraction per round of service. The number of service channels is set to 40 for main rides $(\mathrm{RD}=$ 40), 20 for medium-sized rides (M-RD = 20) and 20 for live shows (LS $=20)$, unless otherwise specified in the figures. The service times are 60 seconds for rides and restaurants, 120 seconds for medium-sized rides, and 300 seconds for live shows. When a mobile node reaches to an attraction, if the queue is full, the mobile node waits nearby the attraction and enters the queue afterwards.

Initially, the mobile nodes are randomly distributed to the fractal points as their start locations. We assume each mobile node has a constant speed of $1 \mathrm{~m} / \mathrm{s}$. Minimum waiting time in a noise point is 30 seconds and Pareto alpha value is empirically set to 1.5 . Lee et al. [12] propose using the least-action trip planning on the waypoints of real-life traces to determine the alpha value in Algorithm 1. The same method is applied to the Disney World GPS traces. The alpha value of 3.0, resulting in the minimum error rate, is used in the simulation study. The flight length difference (error rate) is $1.94 \%$ for $\alpha=3.0$. The error rate becomes $6.01 \%$ for $\alpha=2.5,22.57 \%$ for $\alpha=2$, and $58.22 \%$ for $\alpha=1.5$.

\subsection{Simulation results}

We conducted simulation experiments and generated synthetic mobility traces of the theme park (TP) mobility model. The mobility traces are analyzed by comparison with 41 GPS traces from the CRAWDAD archive, which are collected from smartphones of 11 volunteers who spent their Thanksgiving or Christmas holidays in the Walt Disney World parks [17]. The average duration of the mobility traces is approximately 9 hours with a minimum of 2.2 hours and maximum of 14.3 hours. The GPS tracking logs have a sampling time of 30 seconds. We filtered out the data, assuming the visitors are traveling by transportation vehicles 


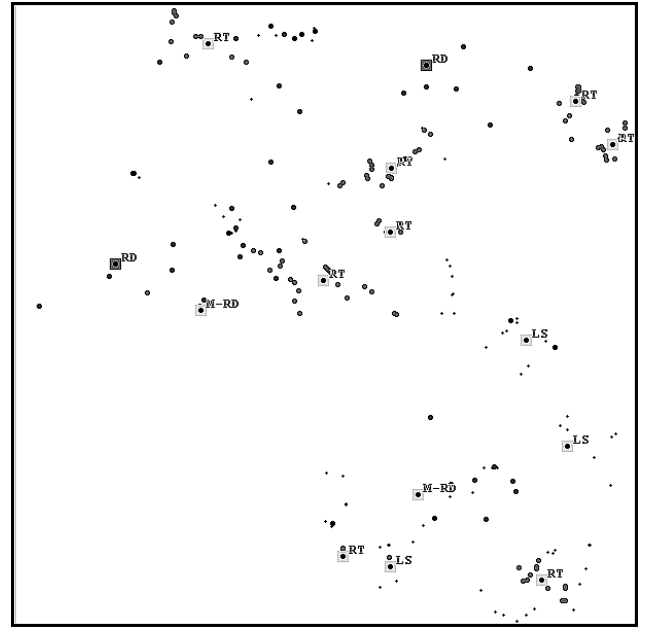

Fig. 8. Positions of 200 mobile nodes after 1 hour simulation time.

when they exceed their regular movement speeds during each sampling time. Moreover, we analyzed the validity of the results by comparing them with synthetic mobility traces of SLAW [12] and RWP [2] mobility model simulations. We examine fundamental characteristics of mobility features, including distribution of flight lengths, average flight lengths, distribution of waiting times, and waiting rate (number of waiting points per hour) of the mobile nodes.

For SLAW and RWP, equally sized areas (1000×1000 meters) are used for the comparison with our model. For the GPS traces, we assume that a visitor is not walking if the visitor moves for more than $150 \mathrm{~m}$ in 30 seconds sampling time, which would exceed the average speed of a person. Accordingly, the data is filtered for the time when the visitors are not walking, but possibly traveling with a bus or another vehicle in the theme park. If a mobile node is in a circular area with a radius of $10 \mathrm{~m}$ in consecutive sampling times of 30 seconds, we assume that the mobile node is waiting in a waiting point.

\subsubsection{Flight lengths}

A flight length is the distance between two consecutive waiting points of a visitor. A waiting point is defined by an attraction or a noise point. Flight length distribution is one of the most significant characteristics of human mobility models since it reflects the scale of the diffusion. Heavy-tail flight lengths in human travels is shown as the characteristic feature of human mobility in several studies ([18], [19]). The flight length distribution results also allow us to make realistic comparisons of human mobility extracted from GPS traces with other mobility models. In this experiment, we compared flight length distribution of the TP mobility model with GPS traces, SLAW and RWP mobility models. The flight length distribution of the GPS traces represents the mobility decision patterns of the real theme park visitors. For instance, the probabilities of theme park visitors to travel to far destination locations (e.g., $400 \mathrm{~m}$ ) as well as their tendency to prefer moving in shorter distances (e.g., 40m) are analyzed with comparisons to the synthetic mobility traces.

The first set of experiments are conducted by using only TP model with the same parameter settings to verify that

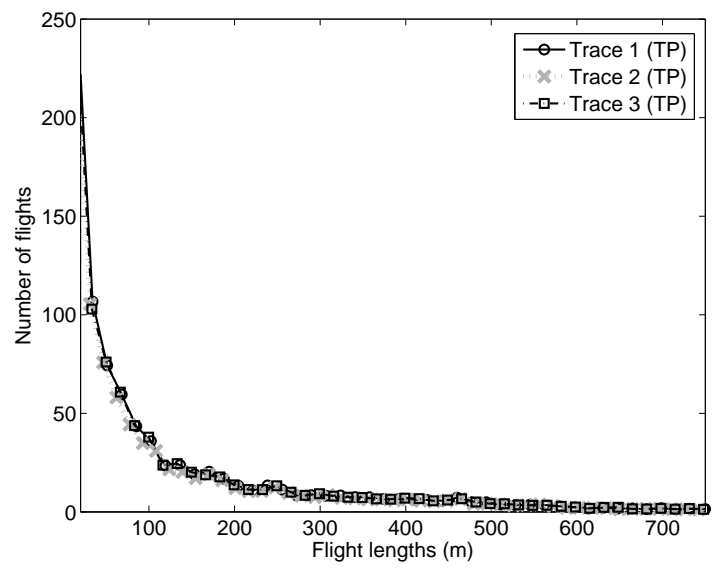

Fig. 9. Flight length distributions of different traces of TP.

the output traces of the simulations are consistent. The flight length distributions of 3 randomly selected experiments are given in Fig. 9. These experiments have flight length counts between 64000 and 68000; however, all the experiments are normalized to the flight length count of 1000. Flight length distributions are consistent, have similar characteristic, and there is no significant difference between the distribution lines. The experiment shows the similar expected outcomes of the synthetic simulation of the mobility model, among different traces of the simulation model.

Next, flight length distribution of the simulation model is compared to GPS traces, SLAW and RWP mobility simulation results. The normalized results of the simulations are given in Fig. 10. The flight length distribution of our model is closer to the GPS traces compared to SLAW or RWP mobility models. SLAW has a similar characteristic but shorter flights and RWP model has a uniform distribution. Fig. 10 also shows that flight length distributions of RWP model is significantly different than the GPS traces. On the other hand, TP and SLAW mobility models represent heavytail flight length distributions.

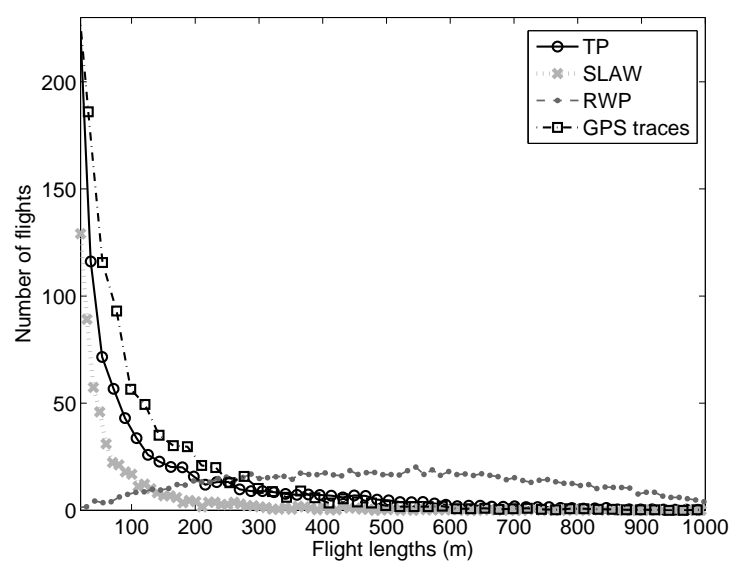

Fig. 10. Flight length distributions of TP, SLAW, RWP, and GPS traces.

Fig. 11 shows the flight length results of TP, SLAW, RWP, and the GPS traces with the confidence bounds for 


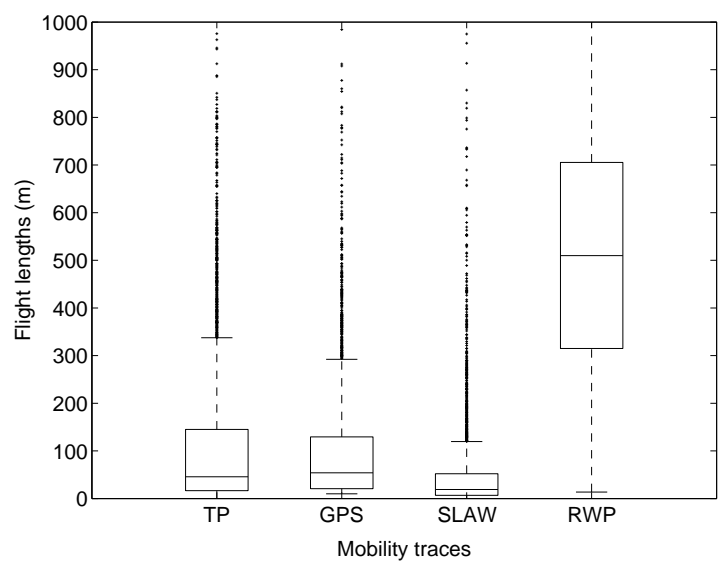

Fig. 11. Flight lengths of TP, GPS traces, SLAW and RWP.

3500 outputs of each trace. The average flight length of the mobility model is very close to the results of GPS traces and the model outperforms the other mobility models. RWP has a very significant difference compared to the other three results. In the 1000x1000 terrain, RWP produced an average value of 500 meters, because of the uniform random selection of next destinations. On the other hand, the flight lengths of SLAW are significantly less than the TP model and GPS traces. In SLAW model, consideration of all fractal points as waiting points produced shorter flights. Table 2 shows the mean, median and standard deviation values of the flight lengths for the mobility models and the GPS traces.

TABLE 2

Flight Length Results

\begin{tabular}{|l|l|l|l|}
\hline & Mean & Median & $\begin{array}{l}\text { Standard } \\
\text { Devia- } \\
\text { tion }\end{array}$ \\
\hline TP & $116.6 \mathrm{~m}$ & $45.7 \mathrm{~m}$ & $163.5 \mathrm{~m}$ \\
\hline GPS & $101.1 \mathrm{~m}$ & $53.9 \mathrm{~m}$ & $132.4 \mathrm{~m}$ \\
\hline SLAW & $51.5 \mathrm{~m}$ & $19.0 \mathrm{~m}$ & $94.6 \mathrm{~m}$ \\
\hline RWP & $500.6 \mathrm{~m}$ & $502.7 \mathrm{~m}$ & $254.1 \mathrm{~m}$ \\
\hline
\end{tabular}

Additionally, we compared the TP traces to analyze the effects of different parameter values on flight lengths. The unique parameters of TP, number of attractions, noise point ratio, and number of service channels of the attractions are used for the analysis.

We start analyzing the effects of the unique parameters. Fig. 12 shows the normalized flight length distributions of TP model with 10, 20 and 30 attractions. We observed that even though the flight lengths do not change dramatically, compared to 10 attractions, the increased number of attractions produced shorter flights for 20 and 30 attractions as the distances between the attractions become shorter.

Noise points represent the waiting points of the visitors between the time of visiting attractions. TP model represents this behavior by giving distant noise points very small probabilities to be chosen as the next destinations, compared to the closer noise points. The effect of the ratio of nonclustered fractal points on flight lengths is shown in Fig. 13. In the case of no noise point $(0 \%)$, the visitors always select an attraction that are distant from each other. Therefore, $0 \%$

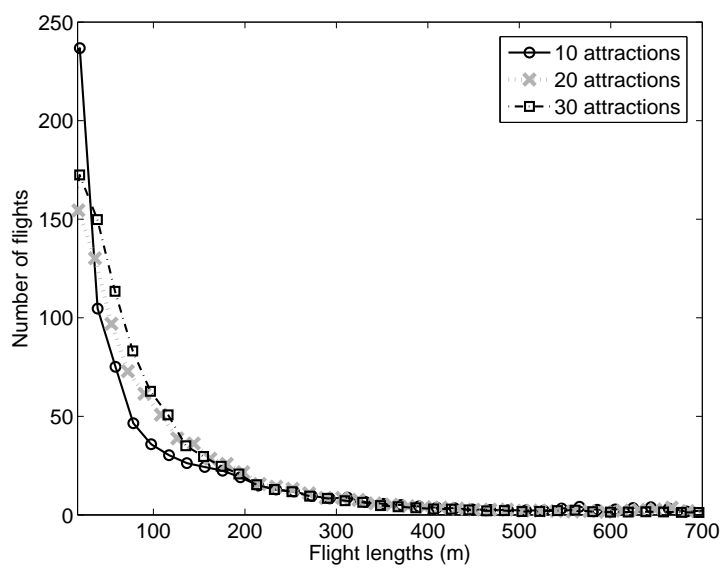

Fig. 12. Flight length distributions of TP with 10, 20, and 30 attractions.

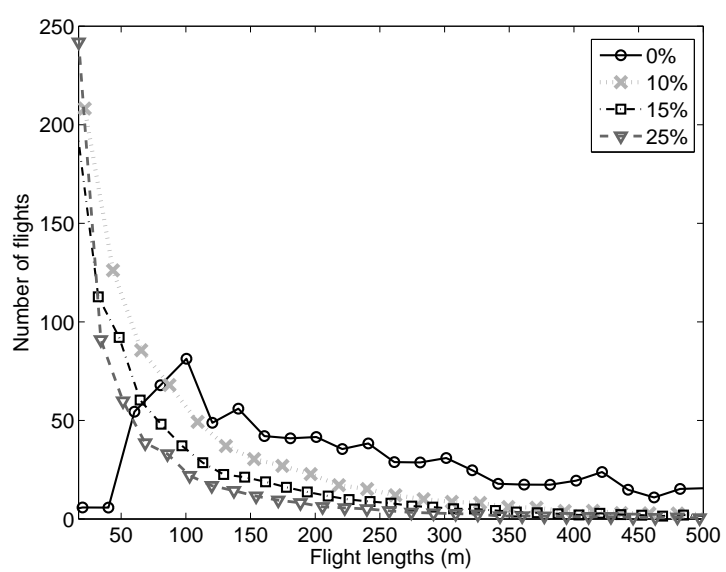

Fig. 13. Flight length distributions of TP with $0 \%, 10 \%, 15 \%$, and $25 \%$ noise point ratios.

noise point ratio produces longer flights. The increase in the noise point ratio causes the increase in the probability of selection of a noise point. Therefore, the increase in noise point ratio shortens the flight lengths of the visitors. The noise point ratio parameter must be configured by using the GPS traces from theme parks for similar mobility traces.

Fig. 14 shows that there is no significant effect of the number of service channels parameter for flight length distributions. The number of service channels effects the waiting time of a visitor in the queue of an attraction, while the waiting time in attraction does not change the selection of the next destination. RD, M-RD, and LS represents the main rides, medium-size rides and live shows respectively.

The number of visitors in theme parks differs according to date and time. If the data for the number of visitors (population size) in different dates is available, the model can be run for each of these dates and the results would reflect the effect of variation in the number of visitors over time. In order to observe the impact of the number of visitors, we conducted simulations of the TP model with various population sizes, ranging from 500 to 10000 .

The increase in the number of visitors causes spending more time on the attractions with the fixed service rates. 


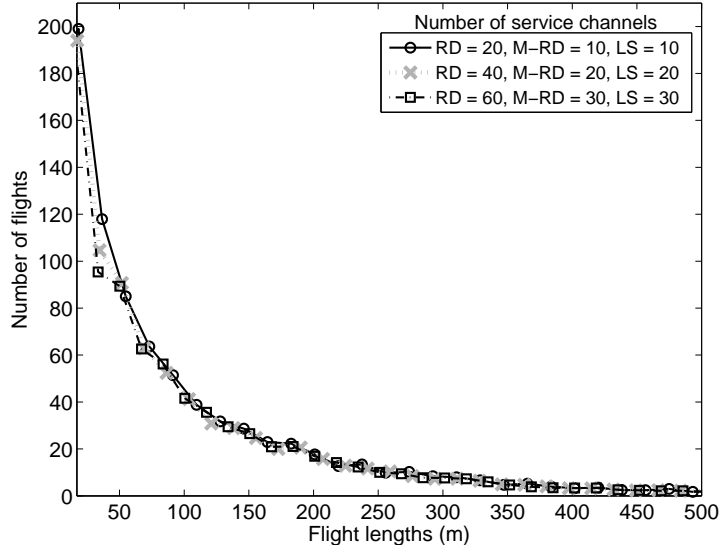

Fig. 14. Flight length distributions of TP with 3 settings for the number of service channels. $(\mathrm{RD}=$ main rides, $\mathrm{M}-\mathrm{RD}=$ medium-size rides, and $\mathrm{LS}$ = live shows)

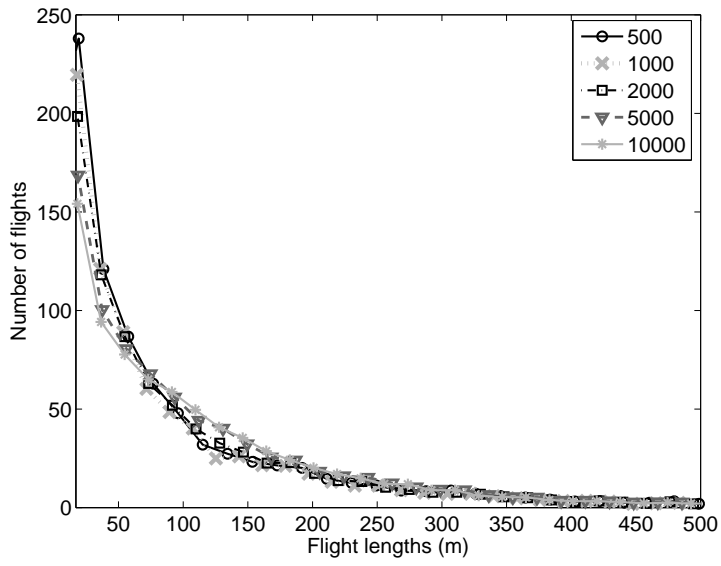

Fig. 15. Flight length distributions of TP with 500, 1000 2000, 5000, and 10000 visitors.

We observe the effect of this parameter on the flight length distributions in Fig. 15. While the parameter does not have a very significant impact on the results, simulations of smaller populations, such as 500, generates traces with shorter flights compared to the larger ones. This is mainly because of spending less time on the attractions. During the hangout times of the 500 visitors, they have extra time to spend in the noise points and traveling to noise points mostly produces shorter flight lengths.

\subsubsection{Number of waiting points}

In this experiment, we analyzed the number of waiting points averaged for one hour for the 3 models and the GPS traces. Average number of waiting points of GPS traces is approximately 10.5 , which means every visitor is waiting at roughly 10 different locations in an hour on average. For SLAW mobility model, the average numbers of waiting points are close to 20 doubling the GPS traces, while it is approximately 7.5 for our simulation and 3.3 for RWP model.

The results of the mobility traces of the models are given in Figure 16. Each trace set includes one trace of

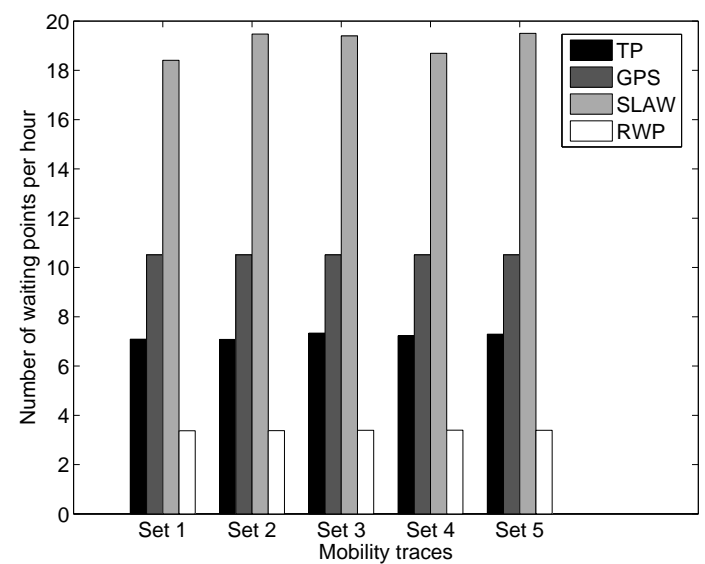

Fig. 16. Number of waiting points per hour for TP, GPS traces, SLAW and RWP for 5 trace sets.

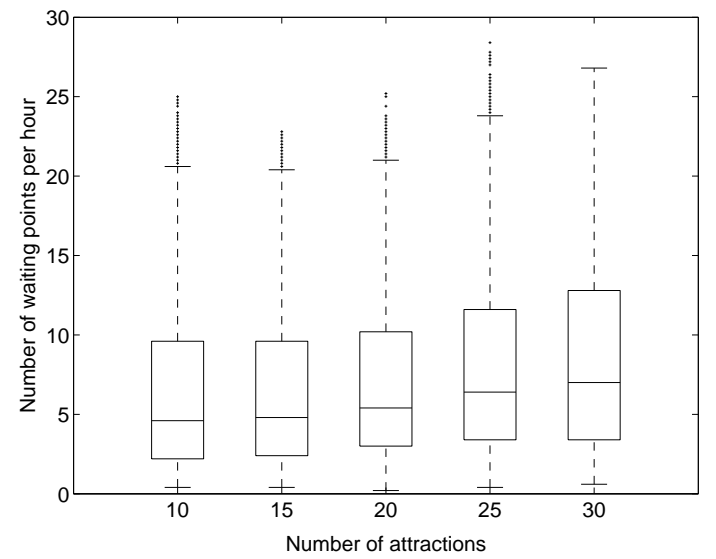

Fig. 17. Number of waiting points per hour for TP with 10, 15, 20, 25, and 30 attractions.

the models. The figure shows that TP model performs significantly better than SLAW and RWP in terms of waiting rates of the mobile nodes. RWP produces very long flights, $500 \mathrm{~m}$ on average, which causes longer times for reaching waiting points. In SLAW model, on the other hand, a mobile nodes moves frequently between 1000 fractal points. TP model combines the behavior of long movements between the attractions, while it allows shorter movements with a probability of visiting noise points. Therefore, TP model is the best match for waiting frequency behavior of theme park visitors.

Furthermore, we analyzed the effect of the parameters on the number of waiting points. In Fig. 17, the increase in the number of attractions slightly increases the number of waiting points since the attractions become closer to each other, the flight lengths become shorter. Thus, the visitors' ability to visit more attractions increases.

Fig. 18 includes the results for noise point ratios ranging from $0 \%$ to $25 \%$. The noise point ratio has a significant impact on the number of waiting points. As the ratio increases from $10 \%$ to $25 \%$, the mean value of the number of waiting points increases approximately by $50 \%$. On the other hand, 


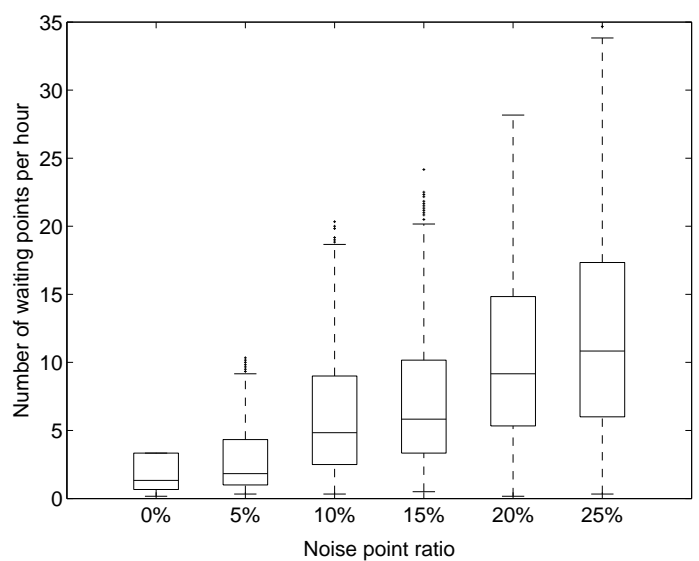

Fig. 18. Number of waiting points per hour for TP with different noise point ratios.

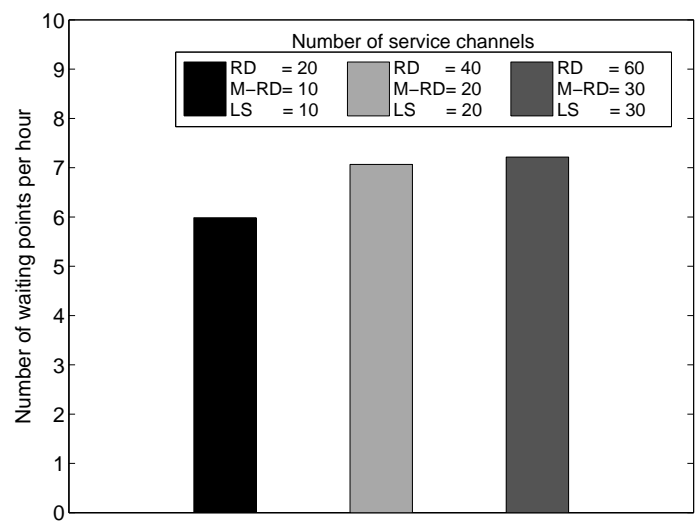

Fig. 19. Number of waiting points per hour for TP with 3 settings of the number of service channels. $(R D=$ main rides, $M-R D=$ medium-size rides, and LS = live shows)

$0 \%$ noise ratio cause the smallest mean value of the number of waiting points. Along with the longer flight lengths, the proportion of the visitors who spend time in attractions becomes higher. This causes longer waiting times in queues for the visitors.

As the number of visitors leaving the attractions in a round of service increases, the waiting time of the visitors in the queues of crowded attractions decreases. As shown in Fig. 19, the number of waiting points per hour increases. However, the effect on the number of waiting points is limited with the decrease of waiting times in the attractions. Higher numbers of service channels in attractions do not produce shorter flights.

Fig. 20 shows the results of the average number of waiting points for the population sizes ranging from 500 to 10000. The population size changes the number of waiting points significantly because it affects the waiting times in the attractions. Average number of waiting points becomes approximately 1.5 per hour for 10000 visitors.

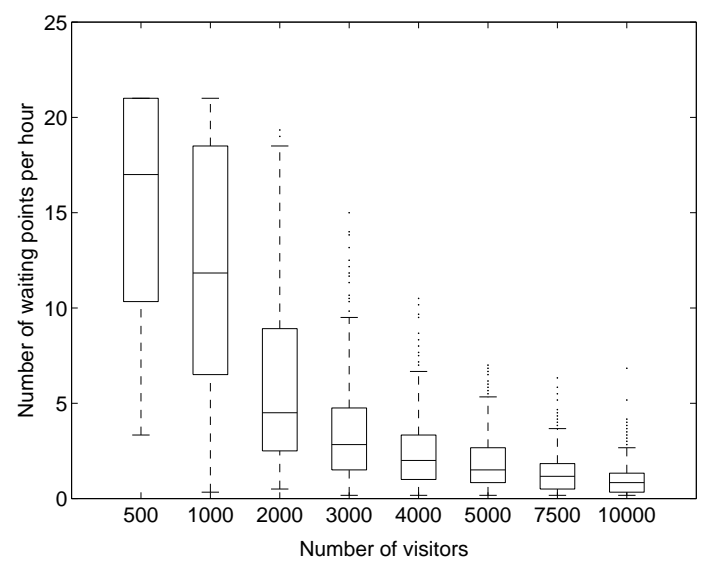

Fig. 20. Number of waiting points per hour for TP with different numbers of visitors.

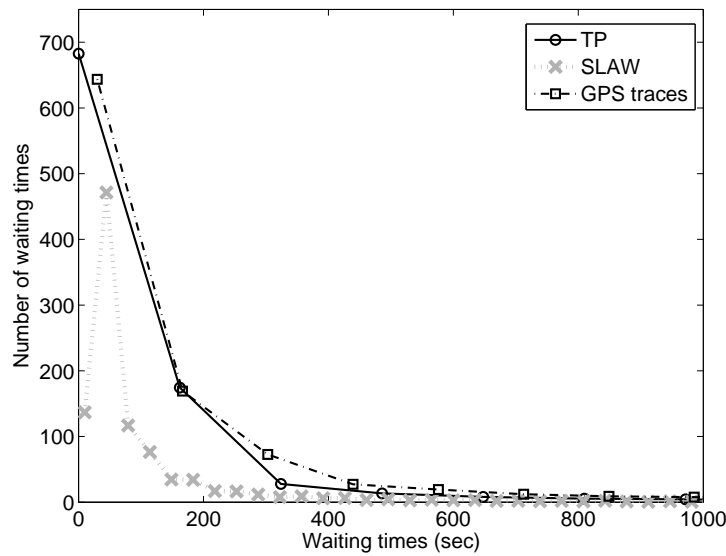

Fig. 21. Waiting time distributions of TP, SLAW, and GPS traces.

\subsubsection{Waiting times}

There are several studies on the waiting times in human walks. These studies ([20], [21]) show that the waiting time distributions have a truncated power-law distribution. While we reflect this in our model for waiting times in noise points by generating the waiting times synthetically, the waiting times in attractions are determined according to the service rates and the number of people in the queues. In this experiment, we compare waiting time distribution of TP with GPS traces and SLAW. Due to constant waiting time of the mobile nodes, we did not include the RWP model in this experiment. The results are normalized to 1000 waiting times.

Fig. 21 shows that waiting time distribution of the proposed model is similar to the GPS traces. Compared to SLAW, TP and GPS traces have shorter waiting times. The results of GPS traces start at 30 seconds, due to the 30 seconds sampling time. By setting noise point ratio, number of attractions, and number of service channels parameters realistically, one can obtain more accurate results to represent the real-world scenario of theme park visitors mobility.

Fig. 22 shows the waiting times of TP model with 10 to 30 attractions. We observed that the number of attractions 


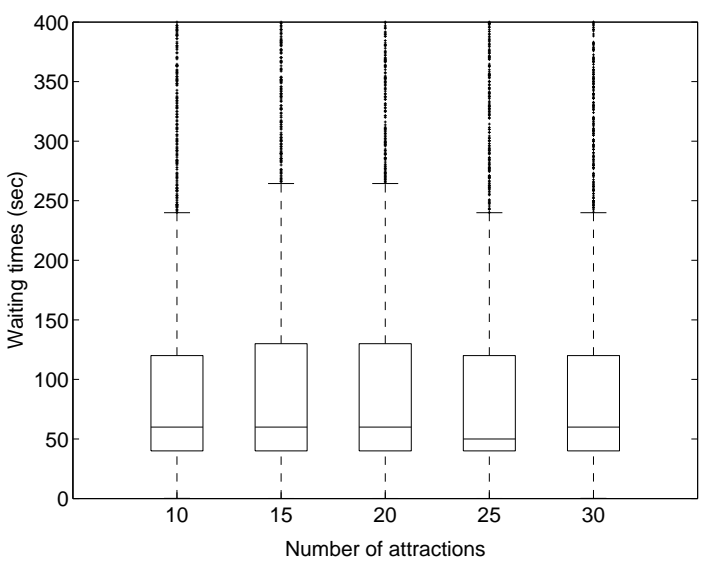

Fig. 22. Waiting times of TP with 10, 15, 20, 25, and 30 attractions.

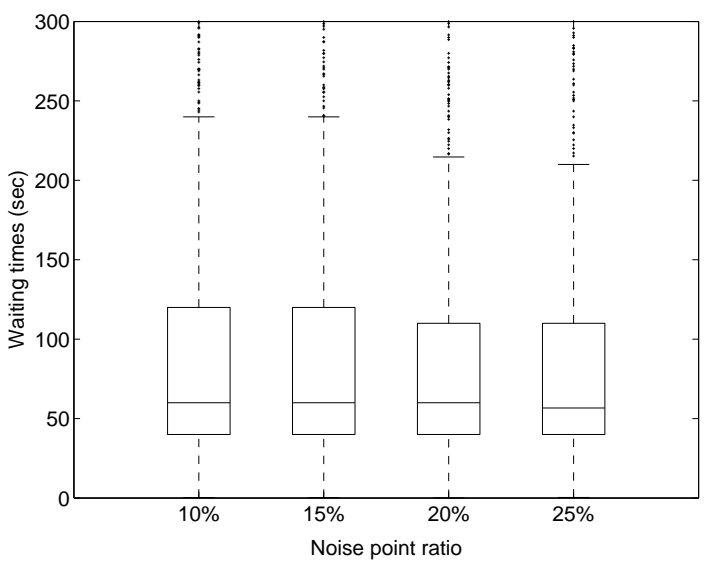

Fig. 23. Waiting times of TP with $10 \%, 15 \%, 20 \%$, and $25 \%$ noise point ratios.

does not have a significant effect on the waiting times as the waiting times stay at approximately the same level. This is due to a tradeoff between visiting more attractions and having less number of people in the queues. Visiting more attractions cause longer average waiting times since the waiting times in noise points is mostly shorter. On the other hand, as the people are distributed to more attractions, fewer people wait in each queue and therefore waiting times in the queues decrease.

As shown in Fig. 23, noise point ratio does not have a significant effect on the waiting times, since the waiting times are mostly effected by the attractions. Still, the waiting times become slightly less because the probability of waiting in a noise point increases. Moreover, the standard deviation becomes smaller. This result shows that variation of the waiting times at noise points is smaller than the variation at attractions. The waiting time in an attraction highly varies because of the number of people waiting in the queue.

Fig. 24 shows the waiting time distributions of TP model with 3 settings of the parameter, the number of service channels. Comparing the first setting which is 20, 10, and 10 for main rides, medium-sized rides, and live shows respectively, with the third setting, it can be seen that waiting times

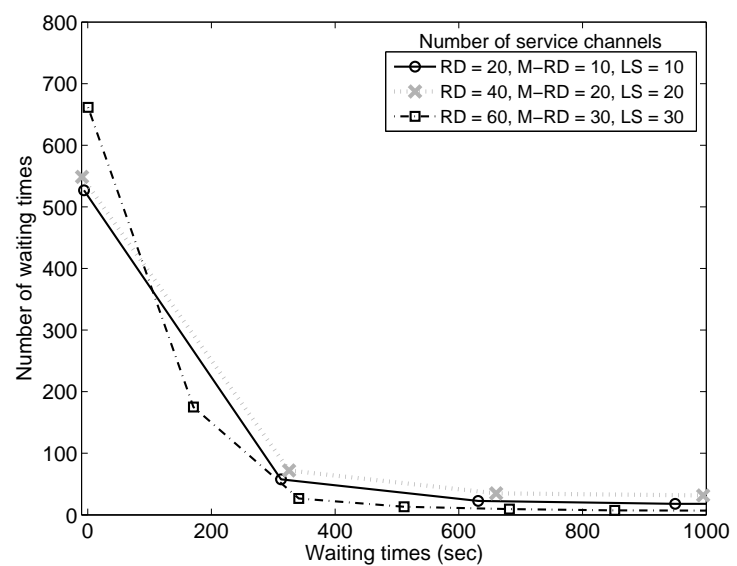

Fig. 24. Waiting time distributions of TP with 3 settings of the number of service channels.

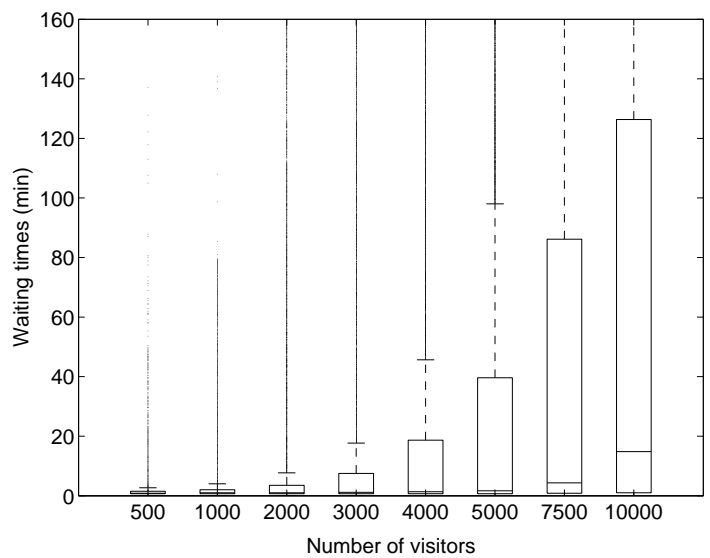

Fig. 25. Waiting times of TP according to number of visitors from 500 to 10000 .

of the first setting is higher than the third one. Since the attractions serve more people with higher numbers of service channels, the waiting times at the attractions decrease. This effect becomes more significant with the decrease in the number of attractions and with the higher numbers of mobile nodes.

The waiting time distributions of our model with respect to the population size is shown in Fig 25. More people in theme park cause longer waiting times, because of sharing the same attractions. As it can be seen in the figure, waiting times increase with the increased population sizes. While attractions do not cause significant waiting times for 500 visitors, they require on average 15 minutes and up to 2 hours waiting times for 10000 visitors. On the other hand, some attractions with less popularity may still not require longer waiting times.

Overall, we observed that the proposed model outperforms SLAW and RWP for the specified metrics, compared to the GPS traces. Moreover, the model gives a consistent performance. Among the tested parameters, noise point ratio is the most effective one, as it has a direct effect on the probability of selecting attractions as the next destinations. 
As expected, population size affects the waiting times and the average number of waiting points since more people cause increase in waiting times at the attractions.

\section{Related Work}

Mobility affects the performance of network applications designed for a group of mobile users or nodes. As the usage of mobile elements in networked systems becomes popular, the effect of mobility becomes critical for various applications such as modern communication systems of urban environments [22] and online services [23]. Considering the impact of mobility, various approaches are proposed for problems including topology control of networks [24], [25], routing in mobile sensor networks [26], [27], [28], tracking in sensor networks [29], [30], [31], analysis of social networks [32], [33], disease spread simulation [34], and opportunistic communication [35]. Hara [36] analyzes the effects of 5 random mobility models on data availability, while Carofiglio et al. [37] use Random Direction Mobility model to provide optimal path selection for routing in mobile ad hoc networks.

Human mobility has several characteristic features, which have been observed by various measurement methods. Instances of these features are truncated power-law distributions of pause times, inter-contact times, fractal waypoints and heterogeneously defined areas of individual mobility. Rhee et al. ([38], [12]) show that these properties are similar to the features of Lévy walks and used these properties to design Self-similar Least Action Walk (SLAW) model. SLAW is a context based Lévy Walk model, which produces synthetic human walk traces by taking the degree of burstiness in waypoint dispersion and heavy-tail flight distribution as inputs. According to SLAW mobility model, the mobile nodes walk from one pre-defined waypoint to another. The dense regions of waypoints form the areas where the people pause and spend most of the time.

SLAW models human mobility in a general context where the waiting times at the waypoints are determined according to a power law distribution. However, for our particular theme park scenario, the waiting times must be defined according to the characteristics of the specific types of attractions. The attractions at theme parks can be combined into groups of main rides, medium-sized rides, live shows and restaurants [15]. Using the specific types, we modeled the waiting times of visitors at these attractions using queueing theoretical models. Basically, in our model, queueing theory is integrated with the visitors' movement decisions, to create a realistic user mobility model for the theme parks. A simple mobility model, SMOOTH [39] is proposed to represent the similar characteristic human mobility features of SLAW model. González et al. [18] analyze the trajectory of 100,000 mobile phone users for a time period of six months and find that the trajectories have a high degree of temporal and spatial regularity. Social force model [40] is proposed by Helbing and Johansson to represent the micro-mobility behavior of the pedestrians in crowded areas. Song et al. [41] analyzed the mobile phone users trajectories and found a $93 \%$ potential predictability in mobility of the users.
For the modeling of human movement in specific scenarios, a variety of mobility models have been proposed. Liu et al. [42] propose a physics-based model of skier mobility in mountainous regions by considering the physical effects of gravity and the steepness of the terrain. The goal of the model is to evaluate the effectiveness of wireless communication devices in improving avalanche safety. Kim et al. [43] propose a mobility model for urban wireless networks, in which the model parameters are derived from urban planning surveys and traffic engineering research. ParkSim [44] by Vukadinovic et al. is a software tool simulating the mobility of theme park visitors. The mobility model of ParkSim is driven by the possible activities of the visitors in the park. Munjal et al. [45] review the changing trends of the recently proposed mobility models used for simulations of opportunistic communication networks. In [46], we propose a human mobility model for the disaster scenarios in theme parks. We consider the evacuation behavior of theme park visitors during the times of natural or man-made disasters. Since the micro-mobility and dynamics of the pedestrian flows have significant effects on the evacuation time, we focus on using real theme park maps and social force model for modeling human mobility in disasters. In this paper, on the other hand, we aim to provide a statistical match with ordinary visitor movement given basic parameters such as number of people, number of attractions, size of the park, and so on.

\section{CONCLUSION}

In this paper, we present a model for the movement of visitors in a theme park. In this model, we combined the nondeterministic behavior of the human mobility with the deterministic behavior of attractions in a theme park. We divided the attractions into groups of main rides, mediumsized rides, live shows and restaurants. We used queueingtheoretic models to calculate times spent by visitors at different attractions. We validated the accuracy of our model through extensive simulations using theme park statistics, GPS traces collected in Disney World theme parks and the data generated by simulations of other mobility models. The results show that our model provides a better match to the real-world data compared to SLAW and RWP models.

We believe that an important outcome of our work is the generation of realistic mobility traces of theme park visitors for theme parks with various scales. The techniques developed in this paper can be used to model human mobility in places which restrain people from using transportation vehicles. These places include airports, shopping malls, fairs, and festivals. For instance, in airports, travelers usually spend time and walk between the pre-determined places (hot-spots), such as check-in locations, restaurants, gates, and security check points.

By studying human mobility, we learned that the mobility behaviors of people in various environments produce significantly different movement patterns. While networks with human participants are becoming increasingly popular, there is still a need for further research in human mobility models. 


\section{REFERENCES}

[1] T. Camp, J. Boleng, and V. Davies, "A survey of mobility models for ad hoc network research," Wireless Communications and Mobile Computing, vol. 2, no. 5, pp. 483-502, September 2002.

[2] J. Broch, D. A. Maltz, D. B. Johnson, Y.-C. Hu, and J. Jetcheva, "A performance comparison of multi-hop wireless ad hoc network routing protocols," in Proc. of the MobiCom'98, October 1998, pp. 85-97.

[3] R. Groenevelt, E. Altman, and P. Nain, "Relaying in mobile ad hoc networks: The brownian motion mobility model," Wireless Networks, vol. 12, no. 5, pp. 561-571, September 2006.

[4] N. Aschenbruck, A. Munjal, and T. Camp, "Trace-based mobility modeling for multi-hop wireless networks," Computer Communications, vol. 34, no. 6, pp. 704-714, May 2011.

[5] F. Bai and A. Helmy, "A survey of mobility models in wireless ad hoc networks," in Wireless Ad Hoc and Sensor Networks, October 2006, pp. 1-30.

[6] S. Isaacman, R. Becker, R. Cáceres, M. Martonosi, J. Rowland, A. Varshavsky, and W. Willinger, "Human mobility modeling at metropolitan scales," in Proc. of the ACM MobiSys'12, 2012, pp. 239-252.

[7] G. Solmaz, M. Akbas, and D. Turgut, "Modeling visitor movement in theme parks," in Proc. of the IEEE LCN'12, October 2012, pp. 3645.

[8] G. Solmaz and D. Turgut, "Event coverage in theme parks using wireless sensor networks with mobile sinks," in Proc. of the IEEE ICC'13, June 2013, pp. 1522-1526.

[9] G. Solmaz and D. Turgut, "Optimizing event coverage in theme parks," Wireless Networks (WINET) Journal, vol. 20, no. 6, pp. 14451459, August 2014.

[10] G. Solmaz, K. Akkaya, and D. Turgut, "Communicationconstrained p-center problem for event coverage in theme parks," in Proc. of the IEEE GLOBECOM'14, December 2014, pp. 486-491.

[11] C. Papageorgiou, K. Birkos, T. Dagiuklas, and S. Kotsopoulos, "Modeling human mobility in obstacle-constrained ad hoc networks," Ad Hoc Networks, vol. 10, no. 3, pp. 421 - 434, May 2012

[12] K. Lee, S. Hong, S. J. Kim, I. Rhee, and S. Chong, "SLAW: self-similar least-action human walk," IEEE/ACM Transactions on Networking, vol. 20, no. 2, pp. 515-529, April 2012.

[13] I. Rhee, K. Lee, S. Hong, S. J. Kim, and S. Chong, "Demystifying the levy-walk nature of human walks," CSC, NCSU, Tech. Rep., 2008.

[14] M. Ester, H. P. Kriegel, J. Sander, and X. Xu, "A density-based algorithm for discovering clusters in large spatial databases with noise," in Proc. of the ACM SIGKDD'96, August 1996, pp. 226-231.

[15] S. Wanhill, "Theme parks: Their development and operation," in Proc. of the CAUTHE'06, February 2006, pp. 1889-1921.

[16] M. Haklay and P. Weber, "OpenStreetMap: User-generated street maps," Pervasive Computing, vol. 7, no. 4, pp. 12-18, December 2008.

[17] I. Rhee, M. Shin, S. Hong, K. Lee, S. Kim, and S. Chong, "CRAWDAD data set ncsu/mobilitymodels (v. 2009-07-23)," Downloaded from http://crawdad.org/ncsu/mobilitymodels/, July 2009.

[18] M. C. González, C. A. Hidalgo, and A.-L. Barabási, "Understanding individual human mobility patterns," Nature, vol. 453, no. 7196, pp. 779-782, June 2008.

[19] D. Brockmann, L. Hufnagel, and T. Geisel, "The scaling laws of human travel," Nature, vol. 439, no. 7075, pp. 462-465, 2006.

[20] M. Kim, D. Kotz, and S. Kim, "Extracting a mobility model from real user traces." in Proc. of the IEEE INFOCOM'06, vol. 6, 2006, pp. 1-13.

[21] I. Rhee, M. Shin, S. Hong, K. Lee, and S. Chong, "On the levy-walk nature of human mobility," in Proc. of the IEEE INFOCOM'08, April 2008, pp. 1597-1605.

[22] F. P. Tso, J. Teng, W. Jia, and D. Xuan, "Mobility: A doubleedged sword for HSPA networks: A large-scale test on hong kong mobile HSPA networks," IEEE Transactions on Parallel and Distributed Systems, vol. 23, no. 10, pp. 1895-1907, October 2012.

[23] S. Wang, Y. Cui, S. Das, W. Li, and J. Wu, "Mobility in IPv6: Whether and how to hierarchize the network?" IEEE Transactions on Parallel and Distributed Systems, vol. 22, no. 10, pp. 1722-1729, October 2011.

[24] H. Nishiyama, T. Ngo, N. Ansari, and N. Kato, "On minimizing the impact of mobility on topology control in mobile ad hoc networks," IEEE Transactions on Wireless Communications, vol. 11, no. 3, pp. 1158-1166, March 2012.
[25] S. Basagni, D. Turgut, and S. Das, "Mobility-adaptive protocols for managing large ad hoc networks," in Proc. of the IEEE ICC'01, June 2001, pp. 1539-1543.

[26] M. I. Akbas, M. R. Brust, and D. Turgut, "SOFROP: Self-organizing and fair routing protocol for wireless networks with mobile sensors and stationary actors," Computer Communications, vol. 34, no. 18, pp. 2135-2146, December 2011.

[27] R. Rahmatizadeh, S. Khan, A. Jayasumana, D. Turgut, and L. Bölöni, "Routing towards a mobile sink using virtual coordinates in a wireless sensor network," in Proc. of the IEEE ICC'14, June 2014, pp. 12-17.

[28] A. Boukerche, B. Turgut, N. Aydin, M. Ahmad, L. Bölöni, and D. Turgut, "Routing protocols in ad hoc networks: a survey," Computer Networks (Elsevier), vol. 55, no. 13, pp. 3032-3080, September 2011.

[29] D. Turgut, B. Turgut, and L. Bölöni, "Stealthy dissemination in intruder tracking sensor networks," in Proc. of the IEEE LCN'09, October 2009, pp. 22-29.

[30] D. Turgut and L. Bölöni, "A pragmatic value-of-information approach for intruder tracking sensor networks," in Proc. of the IEEE ICC'12, June 2012, pp. 4931-4936.

[31] D. Turgut and L. Bölöni, "IVE: Improving the value of information in energy-constrained intruder tracking sensor networks," in Proc. of the IEEE ICC'13, June 2013, pp. 6360-6364.

[32] M. Akbas, R. Avula, M. Bassiouni, and D. Turgut, "Social network generation and friend ranking based on mobile phone data," in Proc. of the IEEE ICC'13, June 2013, pp. 1444-1448.

[33] E. Bulut and B. Szymanski, "Exploiting friendship relations for efficient routing in mobile social networks," IEEE Transactions on Parallel and Distributed Systems, vol. 23, no. 12, pp. 2254-2265, December 2012.

[34] S. Kopman, M. Akbas, and D. Turgut, "Epidemicsim: Epidemic simulation system with realistic mobility," in Proc. of the IEEE P2MNet'12, October 2012, pp. 663-669.

[35] A. Chaintreau, P. Hui, J. Crowcroft, C. Diot, R. Gass, and J. Scott, "Impact of human mobility on opportunistic forwarding algorithms," IEEE Transactions on Mobile Computing, vol. 6, no. 6, pp. 606-620, June 2007.

[36] T. Hara, "Quantifying impact of mobility on data availability in mobile ad hoc networks," IEEE Transactions on Mobile Computing, vol. 9, no. 2, pp. 241-258, February 2010.

[37] G. Carofiglio, C. Chiasserini, M. Garetto, and E. Leonardi, "Route stability in manets under the random direction mobility model," IEEE Transactions on Mobile Computing, vol. 8, no. 9, pp. 1167-1179, September 2009.

[38] I. Rhee, M. Shin, S. Hong, K. Lee, S. J. Kim, and S. Chong, "On the Lévy-walk nature of human mobility," IEEE/ACM Transactions on Networking, vol. 19, no. 3, pp. 630-643, June 2011.

[39] A. Munjal, T. Camp, and W. C. Navidi, "SMOOTH: a simple way to model human walks," ACM SIGMOBILE Mobile Computing and Communications Review, vol. 14, no. 4, pp. 34-36, November 2010.

[40] D. Helbing and A. Johansson, "Pedestrian, crowd and evacuation dynamics," Encyclopedia of Complexity and Systems Science, vol. 16, no. 4, pp. 6476-6495, 2010.

[41] C. Song, Z. Qu, N. Blumm, and A.-L. Barabási, "Limits of predictability in human mobility," Science, vol. 327, no. 5968, pp. 10181021, February 2010.

[42] X. Liu, C. Williamson, and J. Rokne, "Physics-based modeling of skier mobility and avalanche rescue in mountainous terrain," in Proc. of the IEEE LCN'10, October 2010, pp. 645-652.

[43] J. Kim, V. Sridhara, and S. Bohacek, "Realistic mobility simulation of urban mesh networks," Ad Hoc Networks, vol. 7, no. 2, pp. 411430, March 2009.

[44] V. Vukadinovic, F. Dreier, and S. Mangold, "Impact of human mobility on wireless ad hoc networking in entertainment parks," Ad Hoc Networks, vol. 12, no. 0, pp. 17 - 34, 2014.

[45] A. Munjal, T. Camp, and N. Aschenbruck, "Changing trends in modeling mobility: a simple way to model human walks," Journal of Electrical and Computer Engineering, vol. 2012, pp. 1-16, October 2012.

[46] G. Solmaz and D. Turgut, "Theme park mobility in disaster scenarios," in Proc. of the IEEE GLOBECOM'13, December 2013 pp. 377-382.

Gürkan Solmaz is currently working toward the $\mathrm{PhD}$ degree in Computer Science from the Department of Electrical Engineering and Computer Science, 
University of Central Florida (UCF). He received his MS degree in Computer Science from University of Central Florida and his BS degree in Computer Engineering from Middle East Technical University (METU), Turkey. His research interests include mobility modeling, coverage in mobile wireless sensor networks, and disaster resilience in networks. He is a student member of the IEEE and ComSoc.

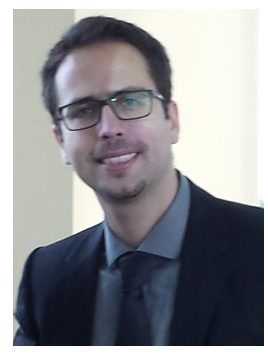

Mustafa İlhan Akbaş received his $\mathrm{PhD}$ degree in Computer Engineering from the Department of Electrical Engineering and Computer Science, University of Central Florida (UCF). He received his BS and MS degrees at the Department of Electrical and Electronics Engineering, Middle East Technical University (METU), Turkey. His research interests include wireless networking and mobile computing, urban sensing and social networks. He is a member of IEEE, ComSoc and IoT.

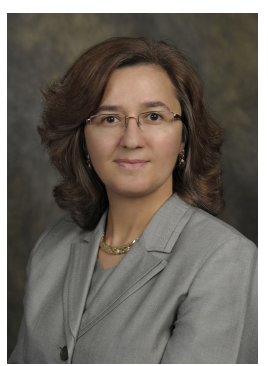

Damla Turgut is an Associate Professor at the Department of Electrical Engineering and Computer Science of University of Central Florida. She received her BS, MS, and PhD degrees from the Computer Science and Engineering Department of University of Texas at Arlington. Her research interests include wireless ad hoc, sensor, underwater and vehicular networks, as well as considerations of privacy in the Internet of Things. She is also interested in applying big data techniques for improving STEM education for women and minorities. Her recent honors and awards include being selected as an iSTEM Faculty Fellow for 2014-2015 and being featured in the UCF Women Making History series in March 2015. She was co-recipient of the Best Paper Award at the IEEE ICC 2013. Dr. Turgut serves as a member of the editorial board and of the technical program committee of ACM and IEEE journals and international conferences. She is a member of IEEE, ACM, and the Upsilon Pi Epsilon honorary society. 